\title{
A randomized controlled trial of internet- delivered cognitive behaviour therapy for adolescent anxiety disorders in a routine clinical care setting with and without parent sessions
}

Article

Accepted Version

Waite, P., Marshall, T. and Creswell, C. (2019) A randomized controlled trial of internet-delivered cognitive behaviour therapy for adolescent anxiety disorders in a routine clinical care setting with and without parent sessions. Child and Adolescent Mental Health, 24 (3). pp. 242-250. ISSN 14753588 doi: https://doi.org/10.1111/camh.12311 Available at https://centaur.reading.ac.uk/81278/

It is advisable to refer to the publisher's version if you intend to cite from the work. See Guidance on citing.

To link to this article DOI: http://dx.doi.org/10.1111/camh.12311

Publisher: Wiley

All outputs in CentAUR are protected by Intellectual Property Rights law, including copyright law. Copyright and IPR is retained by the creators or other copyright holders. Terms and conditions for use of this material are defined in the End User Agreement. 


\section{www.reading.ac.uk/centaur}

\section{CentAUR}

Central Archive at the University of Reading

Reading's research outputs online 
A randomized controlled trial of internet-delivered cognitive behaviour therapy for adolescent anxiety disorders in a routine clinical care setting with and without parent sessions

Polly Waite ${ }^{\mathrm{a}}$, Tamsin Marshall ${ }^{\mathrm{b}}$ and Cathy Creswell ${ }^{\mathrm{a}}$

a School of Psychology and Clinical Language Sciences, University of Reading, U.K.

${ }^{\mathrm{b}}$ CAMHS Anxiety \& Depression Pathway, Berkshire Healthcare Foundation Trust, School of Psychology and Clinical Language Sciences, University of Reading, U.K.

Corresponding author:

Dr Polly Waite

School of Psychology and Clinical Language Sciences

University of Reading, RG6 6AL

p.l.waite@ reading.ac.uk

Tel: 01183785534

Fax: 01183786665

\section{Conflict of interest statement}

The authors declare no conflict of interest. 


\begin{abstract}
Background: Computerized treatments have been shown to be effective in young people with anxiety disorders within research settings. The aims of this study were to evaluate a selfcompleted, therapist-supported online treatment for adolescent anxiety disorders in a routine clinical care setting, and examine whether additional sessions for parents improved treatment outcome.
\end{abstract}

Method: 60 adolescents (13-18 years) referred by primary and secondary care services for treatment of an anxiety disorder and their parent(s) were randomly allocated to begin treatment immediately or after a 16-week waitlist. Half the parents (receiving treatment immediately or after a waitlist) were allocated to receive sessions themselves. Assessments were conducted pre- and post-treatment and at 6-month follow-up.

Results: There was no significant difference post-treatment between the immediate treatment and waitlist groups in remission of primary anxiety disorder (Odds Ratio $(\mathrm{OR})=2.19,95 \%$ CI 0.72-6.70). Parent sessions did not significantly improve adolescent outcomes immediately or at 6-month follow-up $(\mathrm{OR}=0.75,95 \% \mathrm{CI} 0.26-2.15 ; \mathrm{OR}=1.14,95 \% \mathrm{CI}$ $0.42-3.15)$.

Conclusions: Within a routine clinical care setting, a therapist-supported online treatment failed to deliver significantly better outcomes for adolescents with anxiety disorders than a waitlist. Further research is needed to develop more effective treatments for this population. 


\section{Key Practitioner Messages}

- There is a need for studies to evaluate the effectiveness of online treatments for adolescent anxiety disorders in routine clinical care rather than research settings and to explore whether the provision of additional sessions for parents improves treatment outcomes.

- Although around half the adolescents with anxiety disorders seen within a routine clinical care setting (CAMHS) were free of their primary anxiety disorder six months after completing the program, online CBT was not significantly more effective than a waitlist condition and therefore at this stage, its use in routine clinical services is premature.

- Further research is required to examine treatment acceptability among adolescents, and to establish how to optimize outcomes from online treatments in routine care settings.

- Parent sessions did not provide significant additional benefit and it was apparent that parents typically had some level of involvement in their child's treatment in an informal manner, suggesting that the specific parent sessions offered may have been unnecessary. 


\section{Introduction}

Anxiety disorders commonly first occur in early adolescence, with a median age of onset of 11 years (Kessler et al., 2005), and are among the most frequently occurring mental health difficulties in young people (Polanczyk, Salum, Sugaya, Caye, \& Rohde, 2015). If left untreated, they are associated with significant lifelong costs in terms of increased risks of subsequent anxiety, depression, illicit drug dependence, educational underachievement and lower earnings (Knapp, King, Healey, \& Thomas, 2011; Woodward \& Fergusson, 2001). This highlights the importance of effective and accessible interventions for adolescents with anxiety disorders.

Fewer than one in five adolescents with an anxiety disorder receive treatment (Merikangas et al., 2011) and when they do, there can often be a long wait within routine clinical services (Frith, 2016). Low-intensity versions of CBT have been developed that can be delivered by non-specialists in order to improve access to effective interventions. Recently, a number of CBT treatments for children with anxiety disorders have been made available online (e.g., Khanna \& Kendall, 2010; March, Spence, \& Donovan, 2009) and this approach has been found to be effective at a comparable level to clinic-delivered CBT for reducing anxiety (Rooksby, Elouafkaoui, Humphris, Clarkson, \& Freeman, 2015). Adolescent versions of these child treatments have subsequently been developed to be delivered with therapist support using a CD-ROM (Wuthrich et al., 2012) or online (Spence et al., 2011). Wuthrich et al. (2012) compared a computerized program, Cool Teens, to a waitlist control in 43 adolescents aged 14-17 years with a primary anxiety disorder. Adolescents using the computerized program showed significantly greater improvements than those receiving the waitlist control in anxiety diagnoses and symptoms, which were maintained at 3-month follow-up. Remission of primary diagnoses for the adolescents who received the computerized program was $41 \%$ post-treatment and $26 \%$ at 3 -month follow-up, 
whereas all the adolescents on the waitlist continued to meet diagnostic criteria for their primary anxiety disorder at the end of the waitlist. Spence et al. (2011) compared their program, BRAVE for Teenagers-ONLINE, to face to face CBT sessions with a therapist and a waitlist control among 115 adolescents aged 12-18 years with a primary anxiety disorder and their parent(s). There were no significant differences in treatment outcome between the adolescents who completed the program online or in face to face sessions and both treatment groups reported significantly greater reductions in anxiety diagnoses and symptoms compared to the waitlist control. These improvements were maintained at 6 and 12-months follow-up, with $78 \%$ of adolescents who completed the online program (and $80.6 \%$ of those who had face to face sessions) no longer meeting criteria for their primary anxiety disorder one year later. Whilst these results are encouraging, Rooksby et al. (2015) highlight the limitation that studies of computerized CBT have relied largely on self-referral or nonsystematic selection by specialist research clinics, and therefore results may not generalize to routine clinical services, where young people may present with comorbid mood disorders or other difficulties, such as school non-attendance.

Adding online parent sessions alongside sessions for the young person seems intuitively appealing in order to educate parents about CBT, and enable them to support their child during treatment and once sessions come to an end. The relative contribution of parent sessions has been extensively evaluated in studies of children or groups of children and adolescents combined (e.g., Barrett, Rapee, \& Dadds, 1996; Bodden et al., 2008), and metaanalyses have concluded that adding parent sessions to individual child-focused CBT does not in fact improve treatment outcome (e.g., James, James, Cowdrey, Soler, \& Choke, 2013). However online treatments for adolescent anxiety disorders have typically included some parental component. For example, Cool Teens involves adolescents being supported by their parent (to an extent decided by the adolescent), therapist phone calls to the parent (and 
adolescent) and brief parent handouts (Wuthrich et al., 2012), and BRAVE for TeenagersONLINE involves parents completing five online parent sessions and two online booster sessions (Spence et al., 2011). The addition of parental components has not yet been evaluated in studies of treatments aimed specifically at adolescents with an anxiety disorder or in relation to online treatment programs. It is possible that parental sessions may be particularly important in this context, as young people may need further support as they have less direct contact with a therapist than in traditional CBT. On the other hand, the online format may mean that parents can access information from their child's sessions easily, reducing the need for dedicated parent sessions. Given the need to maximize healthcare resources, it will be important to establish whether additional sessions for parents of adolescents with anxiety disorders are worth the additional investment for families, clinicians and services.

The aims of this study were to (a) evaluate the effectiveness of a self-completed, therapist-supported online treatment (BRAVE for Teenagers-ONLINE) for adolescent anxiety disorders in a routine clinical care setting, and (b) explore whether the provision of additional sessions for parents improved adolescent treatment outcome. On the basis of the existing literature, the hypotheses were as follows:

1. Compared with adolescents in a waitlist condition, adolescents receiving BRAVE for Teenagers-ONLINE immediately will be significantly more likely to:

a. Have recovered from their primary anxiety disorder post-treatment [Primary Outcome].

b. Have recovered from all anxiety disorder diagnoses and be much/very much improved on a global index of improvement post-treatment.

c. Have significantly lower levels of clinician-rated, self and parent-report anxiety symptom severity, interference and impairment post-treatment. 
2. Treatment gains will be maintained at six-month follow-up.

We also aimed to explore whether there were significant differences in primary and secondary clinical outcomes in adolescents whose parent(s) completed additional parent treatment sessions, compared with adolescents whose parent(s) did not receive any treatment. Finally, because online interventions have been considered as potential candidates for firststep approaches within a stepped care model of treatment delivery (Andersson, 2009), we also examined the number of young people who were 'stepped up' to further treatment after receiving BRAVE for Teenagers- ONLINE.

\section{Method}

\subsection{Participants}

Participating adolescents were referred to the Berkshire Healthcare NHS Foundation Trust (BHFT) Child and Adolescent Mental Health Service (CAMHS) Anxiety and Depression Pathway for the treatment of anxiety. Recruitment to the study was from July 2012 to December 2013 and all follow-up assessments were completed by December 2014 .

Participants were 60 adolescents aged between $13-18$ years $(\mathrm{M}=14.7, S D=1.42)$ and their parent(s). To be eligible for the trial, adolescents had to meet DSM-IV diagnostic criteria (American Psychiatric Association, 2000) for either generalized anxiety disorder, separation anxiety disorder, social anxiety disorder, specific phobia, panic disorder with or without agoraphobia or agoraphobia without panic disorder, and this had to be identified as the primary problem. Those with primary OCD were excluded from the trial. Participants' diagnoses and demographic information can be found in Table 1. If adolescents were receiving psychotropic medication, they could be included as long as they had been on a stable dosage for two months and agreed to remain on this dosage for the trial. The parent identified as their primary caregiver had to agree to participate in the study and not have a 
significant intellectual impairment. Adolescents were excluded if they had psychotic symptoms, substance dependence, conduct disorder, an autistic spectrum disorder, learning problems that would interfere with their understanding and participation in the trial (based on school, clinic or parent information), had self-harmed within the previous month, were not able to understand and speak English at an age-appropriate level or were currently receiving any other therapy or treatment for anxiety. Participants were also required to have a computer and Internet access at home.

\subsection{Measures}

Details of each measure and information about reliability can be found in Appendix A. Supplementary data. Adolescents' diagnostic assessments were conducted by the assessor and diagnoses were determined using the Anxiety Disorders Interview Schedule (ADIS-C/P) for DSM-IV (Silverman \& Albano, 1996), based on the separate reports of the parent and young person. Overall inter-rater reliability for the assessor team on the ADIS-C/P was excellent (adolescent-report diagnosis: $\mathrm{kappa}=0.95 ; \mathrm{CSR}: \mathrm{ICC}=0.99$; parent-report diagnosis: kappa $=0.94 ;$ CSR: $\mathrm{ICC}=1.00$ ). Adolescents' overall clinical improvement was determined by the assessor using the Clinical Global Impression - Improvement scale (CGII) (Guy, 1976). Overall interrater reliability on the CGI-I was good $(\mathrm{ICC}=.82)$. Overall functioning and impairment was determined by the assessor using the Children's Global Assessment Scale (CGAS) (Shaffer et al., 1983) and the Child Anxiety Impact Scale (CAISC/P) (Langley, Bergman, McCracken, \& Piacentini, 2004). For the CGAS, overall interrater reliability was excellent (ICC $=.93$ ). Symptoms of anxiety and depression were assessed using the Spence Children's Anxiety Scale (SCAS-C/P) and the Short Mood and Feelings Questionnaire (SMFQ-C/P) (Angold et al., 1995). The young person and their parent (reporting on their child) both completed the CAIS, SCAS and SMFQ. Internal consistency for all self-report scales was good to excellent across assessment time points (CAIS-C $\alpha=$ 
.85-.99; CAIS-P $\alpha=.93-.95 ;$ SCAS-C $\alpha=.86-1.00$; SCAS-P $\alpha=.81-.95$; SMFQ-C $\alpha=.90-$

.99; SMFQ-P $\alpha=.93-1.00)$. Adolescents and their parents were given a measure of treatment satisfaction. Finally, parental involvement in adolescent's sessions was determined by asking parents (across both arms) to complete questions post-intervention to investigate how involved they were in their adolescent's treatment sessions.

\subsection{Treatment}

\subsubsection{Online CBT}

The online treatment consisted of BRAVE for Teenagers-ONLINE (Spence, Holmes, Donovan, \& Kenardy, 2006). Sessions incorporate the following standard CBT anxiety management strategies: psychoeducation, relaxation training, recognition of the physiological symptoms of anxiety, cognitive strategies of coping self-talk and cognitive restructuring, graded exposure and problem solving. Parent sessions cover the same material, as well as parenting strategies to enable them to help their adolescents to put into practice anxiety management skills and deal with anxiety-provoking situations (Spence et al., 2011). Further details of the intervention can be found in Appendix A. Supplementary data.

\subsubsection{Waitlist}

The waitlist group did not have any form of planned contact during the waitlist period. The waitlist period was designed to be the same length as the intervention (i.e., 10 weeks $)^{1}$. Once this period was over, participants were re-assessed and then received the online treatment.

\footnotetext{
${ }^{1}$ Although the protocol specified the waitlist period as 10 weeks, this was amended (i.e. the post-treatment assessment for the waitlist group occurred later than originally planned) in
} 


\subsection{Ethical approval and consent}

Ethical approval for the study was granted by the National Research Ethics Committee London - Brent (reference: 12/LO/0119) and the University of Reading Research Ethics Committee, and the trial was registered with ISRCTN (trial registration number: ISRCTN79652741). Written consent was provided from parents. Young people aged 13-15 years provided written assent and those aged 16-18 years provided written consent. .

\subsection{Procedure}

Participants were all referred by primary and secondary care services to BHFT CAMHS Anxiety and Depression Pathway. As part of the routine CAMHS assessment, they were systematically assessed to establish whether they had an anxiety disorder and/or any other diagnoses and to identify the primary problem. Assessments involved both the adolescent and their parent(s) being seen separately to undertake a diagnostic assessment (of the child/adolescent) using the ADIS-C/P (Silverman \& Albano, 1996). Assessments were carried out by honorary assistant psychologists or trainee clinical psychologists who were trained to reliability, received regular supervision and were blind to treatment group allocation. Adolescents and their parent(s) were also asked to independently complete selfreport measures, reporting on the adolescent's symptoms. If the adolescent met eligibility criteria for the trial, both parents and adolescents were given information sheets about the study. The young person and their parent then met with a member of the clinical team for a further appointment to discuss the results of the assessment, to ascertain their willingness to engage in CBT (e.g., completing sessions and being willing to try new ways of doing things) and for it to be delivered via an online intervention. If they chose to take part in the study,

order to ensure that length of time from pre- to post-assessment did not significantly differ between the two groups. 
both provided informed consent. If they chose not to take part, they were placed on the waiting list for treatment within CAMHS.

Participants were randomized to one of two parallel arms, to receive the programme either immediately (IMM) $(n=30)$ or after a waitlist $(W L)$ period $(n=30)$. For the further exploratory part of the study, participants within the IMM arm of the trial were then randomised to receive sessions with (ADOL+PARENT) $(n=15)$ or without (ADOL-ONLY) $(n=15)$ parent sessions. Similarly, adolescents who received treatment after WL were randomised to ADOL+PARENT $(n=15)$ and ADOL-ONLY $(n=15)^{2}$. Figure 1 shows the participant flow, randomization and withdrawals at each stage of the study. Prior to the recruitment, randomization was conducted using computer generated random numbers, with the allocations in numbered, sealed envelopes that were concealed until the assignment of treatment group. Families were assigned to treatment condition by the assessor who had not been involved in the generation of the random allocation sequence. They were then provided with information about the waitlist or, if allocated to begin treatment immediately, information about the program to enable the adolescent (and parent if applicable) to login for their first online session within the following week. Parents (regardless of whether they were allocated to parent sessions or not) were not given any explicit guidance about how or to what extent they should be involved in the adolescents' sessions in order to replicate what would happen in real life.

The therapists on the trial were all graduate psychologists, trainee clinical psychologists or qualified clinical psychologists who received training and regular supervision from an experienced clinical psychologist (PW), who also regularly reviewed

\footnotetext{
${ }^{2}$ This represented a deviation from the original protocol in which it was 1:1:1 (IMM; IMM + PARENT; WL) in order to maximise power for each of the comparisons (IMM versus WL; ADOL-ONLY versus ADOL+PARENT).
} 
therapists' online responses to ensure a high standard of therapy integrity. Further assessments were then conducted post-treatment (for the waitlist group, both at the end of the waitlist period and after completing the online program) and at 6-month follow-up ${ }^{3}$ and were conducted in the CAMHS clinic or in the family's home, or by telephone if they had difficulty attending the appointment in the clinic.

\subsection{Data analysis}

A power calculation was conducted prior to the beginning of the study with $G^{*}$ Power 3, using data from Spence et al. (2011). Spence et al. found that at 12-week post-treatment, adolescents who were allocated to the online treatment condition were 13.45 times more likely to be free of their primary anxiety disorder than those who received the waitlist control condition (Odds Ratio $(\mathrm{OR})=13.45)$. The results of the power calculation indicated that for a two-group comparison (IMM versus WL) using a Chi-Square test, a total sample size of 39 participants would be necessary to detect a large effect size $(0.58)$ at two-sided $\mathrm{p}<0.05$ with $95 \%$ power. To allow for an estimated $20 \%$ loss to follow-up, the sample size was increased and in total, sixty adolescents were recruited to the trial.

SPSS version 22 was used for all statistical analyses. Prior to analyses, variables were screened for missing data, outliers and normality. In line with the CONSORT statement (Moher et al., 2010), statistical methods were not used to examine whether groups differed at baseline. The primary outcome was assessed by intention to treat, with missing data for categorical variables assumed to represent non-remission. For continuous variables, we conducted sensitivity analyses to assess the potential effect of missing data using multiple imputation methods (Sterne et al., 2009), involving the creation of 20 datasets. The completion rate on the primary outcome at post-treatment was $80.0 \%$ and at 6-month followup, $73.3 \%$. There were no significant differences between non-completers and completers on 
the severity of the primary outcome measure at baseline (post-treatment: $t(58)=1.04, p=.30$ ; 6-month follow-up: $t(58)=1.59, p=.12$ ). Imputation for missing data made no difference to the pattern of results and therefore the data presented are based on recorded data only.

The IMM and WL groups were compared at post-treatment using Chi-square tests to examine diagnostic outcomes (i.e., absence of the primary anxiety disorder, absence of all anxiety disorders, and clinical improvement). The continuous secondary outcome measures (i.e., severity of primary anxiety disorder, anxiety/depressive symptoms, interference and functioning/impairment) were analysed using repeated measures analysis of variance (ANOVA). T-tests were used to determine whether there were significant group differences between IMM and WL once both groups had completed the online program, and as there were no significant differences between the IMM and WL groups on all measures except parent-reported anxiety symptoms (see Appendix A. Supplementary data Table 1), data from both groups was combined to examine whether improvements were maintained at 6-month post-intervention using an exact McNemar's test. Comparisons between ADOL+PARENT and ADOL-ONLY at pre-treatment, post-intervention and at 6-month follow-up were conducted using Chi-square and repeated measures ANOVA. Finally, because four participants (13.3\%) from the WL group no longer met criteria for any anxiety disorders at the end of the WL period, further sensitivity analyses were undertaken by repeating all analyses post-intervention and at 6-month follow-up excluding these participants. As this did not change the results, analyses are reported with these participants included. 


\section{Results}

\subsection{Baseline characteristics}

Adolescents' baseline characteristics across the IMM and WL groups can be found in Table 1, and across the ADOL+PARENT and ADOL-ONLY groups in Appendix A. Supplementary data Table 3.

\subsection{Treatment completion}

The mean length of time taken by the IMM group from the baseline assessment (prerandomisation) to the post-treatment assessment was 17.78 weeks $(S D=6.94$; range 10-31 weeks). For the WL group the mean WL period was 16.18 weeks $(S D=5.80$, range 9-32 weeks). This difference was not significant $(t(53)=0.93, p=.36)$. Two adolescents withdrew from the study during the waitlist period due to risk of suicide, which had not been present at recruitment. Four adolescents $(13.3 \%)$ no longer met criteria for any anxiety disorders at the end of the WL period, but still elected to complete the program. Of the 58 participants who were set up to login, $46(79.3 \%)$ completed all 10 sessions of the program and $9(15.5 \%)$ completed both booster sessions. Of the 12 adolescents (20.7\%) who did not complete all 10 treatment sessions, nine $(75.0 \%)$ dropped out prior to session 7 (i.e. before getting under way with exposure).

\subsection{Post-treatment anxiety status}

As shown in Table 2, there were no significant differences between the IMM and WL groups post-treatment on the primary outcome measure of diagnostic status for both remission of primary anxiety disorder diagnosis (IMM 40.0\% versus WL 23.3\%) and all anxiety disorder diagnoses (IMM 26.7\% versus WL 13.3\%). 


\subsection{Post-treatment clinical improvement, symptom severity, interference and impairment}

There was a significant difference between the IMM and WL groups in global clinical improvement (CGI-I), with a greater number of those in the IMM group 'much' or 'very much' improved compared to the WL group (IMM 40.0\% versus WL 16.7\%). However, there were no significant time $\mathrm{x}$ group interaction post-treatment differences in assessor-rated severity (CSR) of primary anxiety disorder, or in adolescent and parent-reported anxiety symptoms (SCAS-C/P), interference of anxiety symptoms (CAIS-C/P), depressive symptoms (SMFQ-C/P) and assessor-rated overall impairment (CGAS) (see Table 2).

\subsection{Post-CBT to 6-month follow-up}

As outlined earlier, data from both groups were combined to examine whether improvements were maintained six months after completing the online program. $53.3 \%$ of all participants were free of their primary anxiety disorder, $28.3 \%$ were free of all anxiety disorder diagnoses and $45.0 \%$ showed 'much' or 'very much' global clinical improvement on the CGI-I. As shown in Appendix A. Supplementary data Table 2, the proportion of adolescents in remission of their primary anxiety disorder was significantly greater at the 6month follow-up compared to the post-CBT assessment. However, there were no significant changes over time in relation to all anxiety diagnoses and clinical improvement, nor on any of the continuous measures from post-treatment to 6-month follow-up: severity of primary anxiety disorder (CSR), overall functioning (CGAS), adolescent and parent-reported anxiety symptoms (SCAS-C/P), depression symptoms (SMFQ-C/P), or adolescent and parentreported inference of anxiety symptoms (CAIS-C/P).

\subsection{Treatment satisfaction}

Forty-five adolescents $(75.0 \%)$ completed questions related to treatment satisfaction, and of these, 44 (97.8\%) had completed all 10 sessions of the program. Twenty-seven 
adolescents (45.0\%) were either 'very' or 'extremely' satisfied, 11 (18.3\%) were 'moderately satisfied', 2 (3.3\%) were 'a little satisfied' and 5 (8.3\%) were 'not at all' satisfied. In terms of whether they would recommend the programme to others, 24 (40.0\%) adolescents responded 'definitely', 15 (25\%) responded 'probably', 3 (5.0\%) were 'unsure' and $3(5.0 \%)$ put 'probably not' or 'definitely not'. Forty-three parents (71.7\%) completed treatment satisfaction questions, and of these, $42(97.7 \%)$ of their children had completed all 10 sessions of the programme. Parent data is presented in Appendix A. Supplementary data Table 5, and demonstrates no significant differences in satisfaction between ADOL-ONLY and ADOL+PARENT.

\subsection{Onward referrals for the treatment of anxiety or depression}

Fifteen $(25.0 \%)$ of the adolescents were referred for further treatment of anxiety or for treatment of depression during or after the trial. Of these, two young people withdrew from the trial and were referred for treatment within CAMHS for depression after their mood deteriorated and level of risk increased (one while on the waitlist and the other while completing the program after receiving the waitlist condition). The remaining 13 adolescents had completed at least seven sessions of the online program. Figure 1 shows referrals to other services by group at each stage of the study. Significantly more adolescents who were allocated to ADOL-ONLY treatment were referred on for further treatment than adolescents allocated to ADOL+PARENT (36.7\% versus $\left.13.3 \% ; \chi^{2}(1)=4.36, \mathrm{p}=.04\right)$, but there were no differences between the adolescents who had immediate treatment and those who received treatment after the waitlist $\left(\chi^{2}(1)=0.09, p=.77\right)$. Of the 15 adolescents referred for further input, $11(73.3 \%)$ were put on the waiting list for face-to-face CBT within CAMHS (nine for anxiety and two for low mood), three (aged 17-18 years; $21.4 \%$ ) were referred to adult 
Improving Access to Psychological Treatment (IAPT) NHS services for the treatment of anxiety and one $(7.1 \%)$ young person was referred to a paediatric psychology service for the treatment of anxiety related to a chronic health problem. Of the $45(75.0 \%)$ adolescents not referred for further treatment, $32(71.1 \%)$ completed the program and did not feel they needed further input $(23(51.1 \%)$ had improved on the CGI; 9 (20.0\%) had not improved on the CGI). The remaining 13 (28.9\%) adolescents had withdrawn from the trial and did not want further help.

\subsection{Parental involvement}

\subsubsection{Parent sessions}

As can be seen in Appendix A. Supplementary data Table 4, there were no significant differences between ADOL-ONLY and ADOL+PARENT on any of the measures, immediately post-CBT or at the 6-month follow-up.

\subsubsection{Parent self-reported involvement in adolescent sessions}

Forty-three (71.7\%) parents completed questions about their involvement in the adolescents' treatment sessions (i.e., in addition to the time spent on their own sessions, if applicable). Table 5 (Appendix A. Supplementary data) provides more information about the nature of parental involvement in treatment.

\section{Discussion}

This study set out first, to evaluate the effectiveness of a therapist-supported online treatment for adolescent anxiety disorders in a routine clinical care setting, and second, to explore whether the provision of additional sessions for parents improved adolescent treatment outcome. The hypotheses that adolescents who received the online treatment would 
be significantly more likely than the waitlist group to have recovered from their primary anxiety disorder and all anxiety disorders post-treatment were not supported. There were also no significant differences on any of the other secondary measures of anxiety symptom severity, interference and impairment, apart from global clinical improvement where the IMM group showed significantly greater improvement than the WL group post-treatment. However, consistent with the hypotheses, treatment gains were maintained at six-month follow-up. We also explored parental involvement in treatment. The majority of parents provided some support to their adolescent in completing the programme, regardless of whether they were offered specific parent sessions, and the inclusion of parent sessions did not lead to significant improvements in outcomes (although it was associated with lower rates of onward referral for further input, see below).

The proportion of adolescents who were free of their primary (40.0\%) and all anxiety disorders (26.7\%) post-treatment was broadly consistent with Spence et al.'s (2011) findings at the 12 -week assessment time point (34.1\% and $18.2 \%$ respectively). This demonstrates that when delivered in a routine clinical care setting, on these variables at least, participants' outcomes were generally in line with those seen in research settings. However, unlike Spence and Wuthrich's studies, in the current study $23.3 \%$ in the WL group no longer met diagnostic criteria for their primary anxiety disorder and $13.3 \%$ no longer had any anxiety disorder at the end of the WL period. Improvements at the level seen in this study are not uncommon (e.g., Barrett et al., 1996). It is possible that some of the sources of anxiety for adolescents, such as school work, exams or relationships with peers, may be more problematic at particular points in the academic year and naturally wax and wane over time depending on the nature of current stressors.

The six-month follow-up results for remission of primary diagnosis were also broadly in line with Spence et al. (2011) (53.3\% versus 54.5\%), however many fewer participants in 
the current study were free of all anxiety disorders (28.3\% versus $45.5 \%)$. In the current study, all participants had been referred to a specialist service and parent-reported child anxiety symptom (SCAS-P) total scores at baseline were considerably higher than in Spence et al.'s study $(M=41.86$ compared to 27.43). Other differences from Spence et al. (2011) were the inclusion of adolescents with primary panic disorder and/or agoraphobia and a greater number of participants with comorbid mood disorders (21.7\% compared to $8.6 \%)$. Our sample also included young people who were not attending school regularly (over 10\% of participants; not reported in Spence et al.). It is possible that differences in sample characteristics explain the greater difficulty participants in this study had in generalising improvements across all anxiety disorders, with some perhaps struggling with motivation and/or opportunities to put the strategies they learnt through the program into practice. Despite the majority of the adolescents continuing to have an anxiety disorder six months post-treatment, three quarters did not want further treatment from NHS services. Although around half the adolescents had shown clinical improvement, the other half had either not shown improvement or had withdrawn from the trial and not attended reassessment. It remains unclear to what extent this reflects improvements in symptoms or functioning that are not being picked up on existing measures versus adolescents and their families feeling that their needs are not being met and as a result, disengaging from services.

Our exploratory analysis of parent sessions suggested that additional parent sessions were not associated with improvements in adolescent treatment outcome, immediately posttreatment or at six-month follow-up, consistent with the existing literature (James et al., 2013). We did not guide parents in how they should be involved in their child's sessions and it was of interest that, regardless of parent condition, the majority of parents tended to be aware of the content of the adolescent's sessions and to get involved to some extent. Despite the lack of differences on clinical outcomes, we found that adolescents in the parent sessions 
arm were significantly less likely to be referred for further input following the trial than adolescents where the parent(s) had not received sessions. The reasons for this remain unclear but may reflect wider family benefits from including the parents in treatment.

Limitations of the study include the number of young people $(20.7 \%)$ who did not complete the online program, although notably this compared favourably to Spence et al (2011) where $61 \%$ had not completed the programme by the 12 -week assessment and $43 \%$ by the 12-month assessment. The study was powered on the basis of the large effect size found by Spence et al. (2011) whereas the size of the effects in the current study were generally small. Although studies of the effectiveness of CBT for anxiety in children and young people within community rather than research settings have typically demonstrated medium to large effect sizes, there is some evidence that treatment may lose some of its efficacy when transported to routine care settings (Jonsson, Thastum, Arendt, \& Juul-Sørensen, 2015). It may be that the modest effects found in the current study would still be clinically meaningful, particularly if this format enables treatment to be delivered more efficiently than other treatment approaches. Notably, around two thirds of the young people and their parents reported that they were satisfied with the treatment and would probably/definitely recommend it to others. Although we have conducted qualitative interviews with participants around treatment acceptability, including the findings is beyond the scope of this paper, and so they will be presented elsewhere. The study was under-powered to examine outcomes for individual anxiety disorders and it may be that some disorders respond better to the program than others (e.g., Kerns, Read, Klugman, \& Kendall, 2013). Although the mean duration between baseline assessment and post-treatment/waitlist assessments was similar across arms, this varied considerably between participants from 9 to 32 weeks, despite therapists actively encouraging participants to complete sessions weekly. Participants were predominantly White British from relatively high socio-economic backgrounds, and this is likely to limit 
generalisation to other populations. Finally, the study would have benefitted from a 12-month follow-up assessment point.

In summary, although around half the adolescents with anxiety disorders seen within a routine clinical care setting were free of their primary anxiety disorder six months after completing the program, online CBT was not significantly more effective than a waitlist condition on any measure except post-treatment global clinical improvement. Furthermore, over two thirds of the adolescents still met diagnostic criteria for an anxiety disorder at the six-month follow-up. While the direct of the effects were all in the predicted direction, the effect sizes were smaller than the study was powered to detect. Further research is required to establish how to optimize outcomes from online treatments, investigate factors that may have an impact on treatment outcome, establish whether online treatments have a role to play in a stepped care treatment model for young people with anxiety disorders and understand the reasons for not wanting further treatment when there is no demonstrable clinical improvement. It will be important to establish that first-line treatments, such as this, do not bring negative outcomes associated with disengagement from services and deter people from seeking other help that may be effective. Treatment acceptability is also important to determine and the findings from qualitative interviews undertaken with participants from this trial will be reported elsewhere. Finally, the provision of parent sessions did not provide significant additional benefit and it was apparent that parents typically had some level of involvement in their child's treatment in an informal manner. Further experimental research is necessary to establish whether and which specific parenting factors maintain adolescent anxiety to pinpoint what, if any, parental factors need to be addressed in treatments for adolescents with anxiety disorders. 


\section{Declarations of Interest}

None

\section{Acknowledgments}

We would like to thank the adolescents and their families who took part in this research for their help. We thank Professor Sue Spence, Dr Sonja March and colleagues for making the BRAVE for Teenagers-ONLINE program available for this study. We would also like to thank staff from BHFT CAMHS Anxiety \& Depression Pathway and AnDY who supported this research. Polly Waite was supported by a Medical Research Council (MRC) Clinical Research Training Fellowship (G1002011). This study was supported by the National Institute for Health Research (NIHR) Clinical Research Network. The authors declare no conflict of interest. The research materials can be accessed by contacting the corresponding author. 
Table 1. Adolescents' baseline characteristics across the immediate treatment and waitlist groups $(n=60)$.

$\begin{array}{ll}\text { IMM } & \text { WL } \\ (\mathrm{n}=30) & (\mathrm{n}=30)\end{array}$

Age in years, mean (SD)

$$
14.20(1.38) \quad 15.20(1.30)
$$

Gender (male:female)

$14: 16 \quad 7: 23$

Ethnicity, White British, n (\%)

$28(93.3)$

$27(90.0)$

Socio-economic status, n (\%)

Not recorded

$1(3.3)$

0

Higher professional

$17(56.7)$

$11(36.7)$

$21(70.0)$

Other employed

Unemployed

$1(3.3)$

8 (26.7)

$1(3.3)$

ADIS primary diagnosis, n (\%)

Social anxiety disorder

$9(30.0)$

$7(23.3)$

$10(33.3)$

Generalized anxiety disorder

$6(20.0)$

$8(26.7)$

Specific phobia

$3(10.0)$

$5(16.7)$

Separation anxiety disorder

Panic disorder with agoraphobia

$3(10.0)$

$2(6.7)$

Panic disorder without agoraphobia

$1(3.3)$

$4(13.3)$

Agoraphobia

$1(3.3)$

$1(3.3)$

Total number of anxiety disorders, mean (SD) 1.90 0.76)

$1.57(0.68)$

Presence of other psychiatric disorders, n (\%)

Dysthymia

$6(20)$

$2(6.7)$

$1(3.3)$

Attention-deficit hyperactivity disorder

$1(3.3)$

$4(13.3)$

$1(3.3)$

Oppositional defiant disorder

$4(13.33)$

$1(3.3)$

Presence of school refusal, n (\%)

$1(3.3)$

$3(10)$

Psychotropic medication, n (\%)*

$1(3.3)$

Note. ADIS = Anxiety Disorders Interview Schedule for DSM-IV, IMM = adolescents receiving the intervention immediately after allocation, $\mathrm{n}=$ sample size, $S D=$ standard deviation, $\mathrm{WL}=$ waitlist condition. * One adolescent (WL group) was taking a selective serotonin reuptake inhibitors (SSRI) and the other adolescent (IMM group) was taking a betablocker and a tricyclic antidepressant. 
Table 2. Differences between the immediate treatment and waitlist group on anxiety status, severity, symptoms, impact, functioning and comorbid symptoms at baseline and post-treatment.

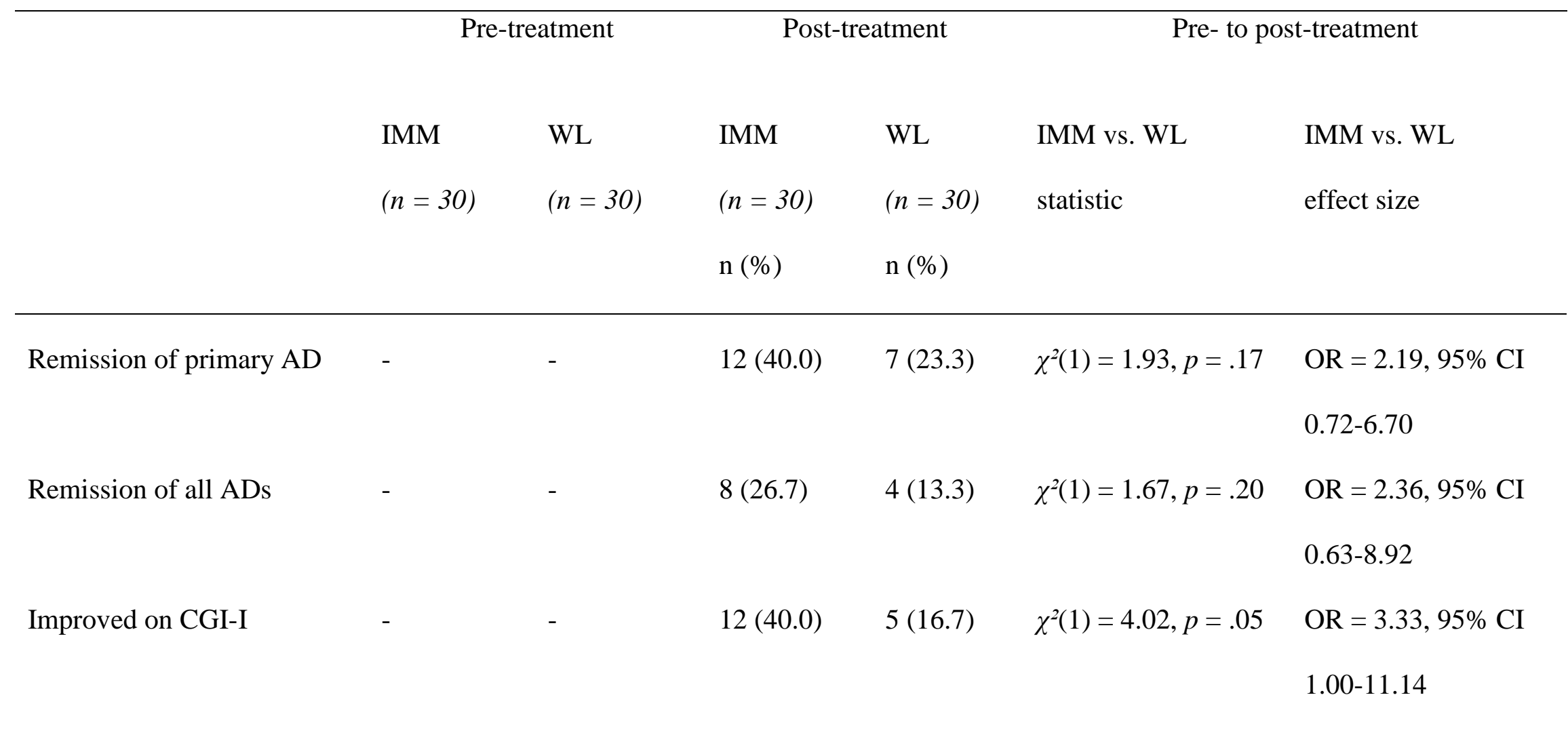




\begin{tabular}{|c|c|c|c|c|c|c|}
\hline \multirow[t]{2}{*}{ CSR } & $5.73(0.87)$ & $5.77(1.01)$ & $3.89(2.58)$ & $4.86(2.19)$ & $F(1,53)=2.53, p=$ & $\eta^{2}=.05,95 \% \mathrm{CI}$ \\
\hline & & & & & .12 & $.00-.19$ \\
\hline \multirow[t]{2}{*}{ CGAS } & $52.30(8.83)$ & $51.90(9.15)$ & 59.48 & 55.18 & $F(1,52)=2.06, p=$ & $\eta^{2}=.04,95 \% \mathrm{CI}$ \\
\hline & & & $(14.87)$ & $(12.48)$ & .16 & $.00-.18$ \\
\hline \multirow[t]{2}{*}{ SCAS-C } & 42.40 & 39.13 & 30.35 & 33.46 & $F(1,52)=2.70, p=$ & $\eta^{2}=.05,95 \% \mathrm{CI}$ \\
\hline & $(16.05)$ & $(14.61)$ & (19.17) & $(15.01)$ & .11 & $.00-.20$ \\
\hline \multirow[t]{2}{*}{ SCAS-P } & 41.86 & 32.27 & 33.12 & 28.93 & $F(1,51)=3.18, p=$ & $\eta^{2}=.06,95 \% \mathrm{CI}$ \\
\hline & $(22.50)$ & $(16.95)$ & $(21.70)$ & $(15.79)$ & .08 & $.00-.21$ \\
\hline \multirow[t]{2}{*}{ CAIS-C } & 22.50 & 22.85 & 18.04 & 17.59 & $F(1,46)=0.33, p=$ & $\eta^{2}=.01,95 \% \mathrm{CI}$ \\
\hline & $(16.83)$ & (13.79) & $(16.97)$ & (13.09) & .57 & $.00-.12$ \\
\hline \multirow[t]{2}{*}{ CAIS-P } & 29.11 & 22.85 & 23.60 & 19.63 & $F(1,46)=1.84, p=$ & $\eta^{2}=.04,95 \% \mathrm{CI}$ \\
\hline & $(21.08)$ & (13.79) & $(21.81)$ & $(16.34)$ & .18 & $.00-.19$ \\
\hline \multirow[t]{2}{*}{ SMFQ-C } & $7.62(5.86)$ & $7.24(5.67)$ & $6.48(6.40)$ & $7.70(7.05)$ & $F(1,44)=0.14, p=$ & $\eta^{2}=.00,95 \% \mathrm{CI}$ \\
\hline & & & & & .71 & $.00-.10$ \\
\hline SMFQ-P & $8.21(7.19)$ & $7.30(6.43)$ & $6.73(6.91)$ & $7.11(7.44)$ & $F(1,44)=0.08, p=$ & $\eta^{2}=.00,95 \% \mathrm{CI}$ \\
\hline
\end{tabular}


Note. $\mathrm{AD}=$ anxiety disorder, CAIS-C/P $=$ The Child Anxiety Impact Scale - child/parent report, CGAS = The Children's Global Assessment Scale, CGI-I = The Clinical Global Impression - Improvement scale, $C I=$ confidence interval, CSR = Clinician Severity Rating on the Anxiety Disorders Interview Schedule for DSM-IV (ADIS), $F=$ analysis of variance (ANOVA) statistic, IMM = adolescents receiving the intervention immediately after allocation, $O R=$ odds ratio (a measure of effect size for use with chi square), $p=$ significance level, $\mathrm{SCAS}-\mathrm{C} / \mathrm{P}=\mathrm{The}$ Spence Children's Anxiety Scale - child/parent report, SMFQ-C/P = The Short Mood and Feelings Questionnaire - child/parent report, WL = waitlist condition, $\eta^{2}=$ eta-squared (a measure of effect size for use with ANOVA), $\chi^{2}=$ chi square statistic. 
Figure 1: Participant flow, randomization, withdrawals and onward referrals at each stage of the study

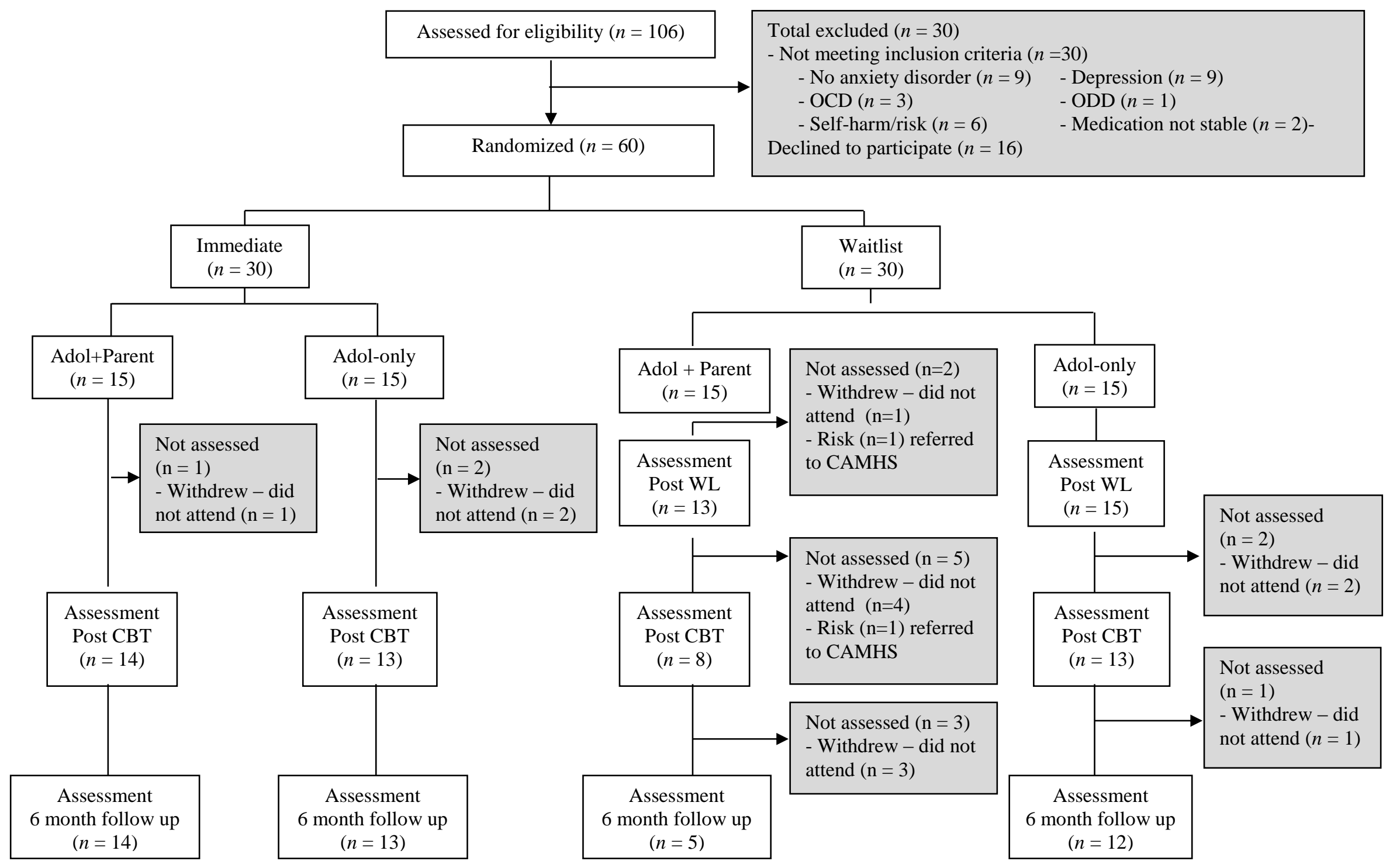




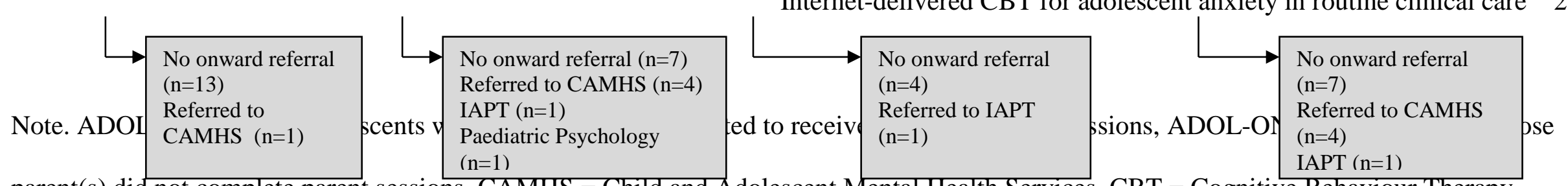

parent(s) did not complete parent sessions, CAMHS = Child and Adolescent Mental Health Services, CBT = Cognitive Behaviour Therapy,

IAPT = Improving Access to Psychological Treatment Adult Service, OCD = Obsessive Compulsive Disorder, ODD = Oppositional Defiant

Disorder, $\mathrm{n}=$ sample size, $\mathrm{WL}=$ waitlist condition. 


\section{References}

American Psychiatric Association. (2000). Diagnostic and statistical manual of mental disorders (Fourth, text rev, ed.). Washington DC: American Psychiatric Association.

Andersson, G. (2009). Using the Internet to provide cognitive behaviour therapy. Behaviour Research and Therapy, 47(3), 175-180.

Angold, A., Costello, E. J., Messer, S. C., Pickles, A., Winder, F., \& Silver, D. (1995). Development of a short questionnaire for use in epidemiological studies of depression in children and adolescents. International Journal of Methods in Psychiatric Research, 5, 237-249.

Barrett, P. M., Rapee, R. M., \& Dadds, M. R. (1996). Family treatment of childhood anxiety: A controlled trial. Journal of Consulting and Clinical Psychology, 64, 333-342.

Bodden, D., Bögels, S., Nauta, M., de Hann, E., Ringrose, J., Appelboom, C., \& Brinkman, K. (2008). Child versus family cognitive behavioural therapy in clinically anxious youth: An efficacy and partial effectiveness study. Journal of Clinical Child and Adolescent Psychology, 47(12), 1384-1394.

Frith, E. (2016). CentreForum Commission on Children and young people's mental health: State of the nation. Centreforum Online at http://centreforum. org/live/wp-content/uploads/2016/04/State-of-theNation-report-web. pdf.

Guy, W. (1976). The Clinical Global Impresssion Scale; ECDEU Assessment Manual for Psychopharmacology. Rockville, MD.

James, A. C., James, G., Cowdrey, F. A., Soler, A., \& Choke, A. (2013). Cognitive behavioural therapy for anxiety disorders in children and adolescents (Review). The Cochrane Library(6). doi:DOI: 10.1002/14651858.CD004690.pub3

Jonsson, H., Thastum, M., Arendt, K., \& Juul-Sørensen, M. (2015). Group cognitive behavioural treatment of youth anxiety in community based clinical practice: clinical significance and benchmarking against efficacy. Journal of Anxiety Disorders, 35, 9-18.

Kerns, C. M., Read, K. L., Klugman, J., \& Kendall, P. C. (2013). Cognitive behavioral therapy for youth with social anxiety: Differential short and long-term treatment outcomes. Journal of Anxiety Disorders, 27, 210-215.

Kessler, R. C., Berglund, P., Demler, O., Jin, R., Merikangas, K. R., \& Walters, E. E. (2005). Lifetime prevalence and age-of-onset distributions of DSM-IV disorders in the national comorbidity survey replication. Archives of General Psychiatry, 62(6), 593-602. doi:10.1001/archpsyc.62.6.593

Khanna, M., \& Kendall, P. C. (2010). Computer-assisted cognitive behavioral therapy for child anxiety: Results of a randomized clinical trial. Journal of Consulting and Clinical Psychology, 78(5), 737745.

Knapp, M., King, D., Healey, A., \& Thomas, C. (2011). Economic outcomes in adulthood and their associations with antisocial conduct, attention deficit and anxiety problems in childhood. Journal of Mental Health Policy and Economics, 14(3), 137-147.

Langley, A. K., Bergman, R. L., McCracken, J., \& Piacentini, J. C. (2004). Impairment in childhood anxiety disorders: Preliminary examination of the child anxiety impact scale-parent version. Journal of Child and Adolescent Psychopharmacology, 14(1), 105-114. doi:10.1089/104454604773840544

March, S., Spence, S. H., \& Donovan, C. L. (2009). The efficacy of an internet-based cognitive-behavioral therapy intervention for child anxiety disorders. Journal of Pediatric Psychology, 34(5), 474-487.

Merikangas, K. R., He, J.-P., Burstein, M., Swendsen, J., Avenevoli, S., Case, B., . . Olfson, M. (2011). Service Utilization for Lifetime Mental Disorders in U.S. Adolescents: Results of the National Comorbidity Survey-Adolescent Supplement (NCS-A). Journal of the American Academy of Child and Adolescent Psychiatry, 50(1), 32-45.

Moher, D., Hopewell, S., Schulz, K. F., Montori, V., Gøtzsche, P. C., Devereaux, P., . . Altman, D. G. (2010). CONSORT 2010 explanation and elaboration: updated guidelines for reporting parallel group randomised trials. $B M J, 340, \mathrm{c} 869$. 
Polanczyk, G. V., Salum, G. A., Sugaya, L. S., Caye, A., \& Rohde, L. A. (2015). Annual research review: A meta- analysis of the worldwide prevalence of mental disorders in children and adolescents. Journal of Child Psychology and Psychiatry, 56(3), 345-365.

Rooksby, M., Elouafkaoui, P., Humphris, G., Clarkson, J., \& Freeman, R. (2015). Internet-assisted delivery of cognitive behavioural therapy (CBT) for childhood anxiety: systematic review and meta-analysis. Journal of Anxiety Disorders, 29, 83-92.

Shaffer, D., Gould, M. S., Brasic, J., Ambrosini, P., Fisher, P., Bird, H., \& Aluwahlia, S. (1983). A children's global assessment scale (CGAS). Archives of General Psychiatry, 40(11), 1228-1231. doi:10.1001/archpsyc.1983.01790100074010

Silverman, W. K., \& Albano, A. M. (1996). The anxiety disorders interview schedule for DSM-IV-child and parent versions. San Antonio. TX: Psychological Corporation.

Spence, S. H., Donovan, C. L., March, S., Gamble, A., Anderson, R. E., Prosser, S., \& Kenardy, J. (2011). A randomized controlled trial of online versus clinic-based CBT for adolescent anxiety. Journal of Consulting and Clinical Psychology, 79(5), 629-642. doi:10.1037/a0024512

Spence, S. H., Holmes, J., Donovan, C. L., \& Kenardy, J. (2006). BRAVE for Teenagers-ONLINE: An Internet based program for adolescents with anxiety. Brisbane: University of Queensland.

Sterne, J. A., White, I. R., Carlin, J. B., Spratt, M., Royston, P., Kenward, M. G., . . Carpenter, J. R. (2009). Multiple imputation for missing data in epidemiological and clinical research: potential and pitfalls. BMJ, 338, b2393. doi:10.1136/bmj.b2393

Woodward, L. J., \& Fergusson, D. M. (2001). Life course outcomes of young people with anxiety disorders in adolescence. Journal of the American Academy of Child and Adolescent Psychiatry, 40(9), 10861093. doi:10.1097/00004583-200109000-00018

Wuthrich, V. M., Rapee, R. M., Cunningham, M. J., Lyneham, H. J., Hudson, J. L., \& Schniering, C. A. (2012). A Randomized Controlled Trial of the Cool Teens CD-ROM Computerized Program for Adolescent Anxiety. Journal of the American Academy of Child and Adolescent Psychiatry, 51(3), 261-270. doi:10.1016/j.jaac.2011.12.002 